C. Dierckxsens, K. Srivastava, M. Reza, S. Cole, J. Beerten, and R. Belmans, "A Distributed DC voltage control method for VSC MTDC systems," Electric Power Systems Research, vol. 82, no. 1, pp. 54-58, Jan. 2012.

Digital Objective Identifier: 10.1016/j.epsr.2011.08.006

URL:

http://www.sciencedirect.com/science/article/pii/S0378779611001933

NOTICE: this is the author's version of a work that was accepted for publication in Electric Power System Research. Changes resulting from the publishing process, such as peer review, editing, corrections, structural formatting, and other quality control mechanisms may not be reflected in this document. Changes may have been made to this work since it was submitted for publication. A definitive version was subsequently published in Electric Power System Research, vol. 82, no. 1, Jan. 2012. 


\title{
A Distributed DC Voltage Control Method for VSC MTDC Systems
}

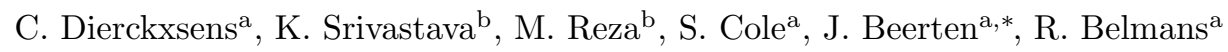 \\ ${ }^{a}$ K.U.Leuven, Division of Electrical Energy and Computer Architectures (ESAT-ELECTA), Kasteelpark Arenberg 10, bus \\ 2445, 3001 Leuven, Belgium \\ ${ }^{b}$ ABB Corporate Research, Forskargränd 7, 72178 Västerås, Sweden
}

\begin{abstract}
With the development of VSC HVDC transmission, the realization of the first VSC MTDC grid is coming within reach. An outstanding issue is DC voltage control in MTDC Systems. The easiest way to maintain stable operation is to assign the task of DC voltage regulation to one converter. This paper discusses a control strategy for an extended VSC MTDC grid using a typical DC voltage control on one DC bus, combined with a DC voltage droop characteristic on the other DC buses. The impact of the proposed control structure on the stability of the AC and DC grid is investigated by numeric simulations using the MatDyn software package.
\end{abstract}

Keywords: Voltage Source Converter (VSC), Multi-Terminal HVDC (MTDC), Voltage Droop

\section{Introduction}

Voltage Source Converter High Voltage Direct Current (VSC HVDC) is a promising technology for future grid expansions and offshore applications in particular. It can be used to feed passive networks and can be kept compact, which makes it more suitable to build connections to offshore wind farms and platforms. The feasibility for such applications has already been widely proven by projects all over the world [1],[2]. Furthermore, VSC HVDC features full controllability of the DC network and makes it easier to connect multiple converters to the same DC grid compared to classic Line Commutated Converter (LCC) HVDC. This creates the possibility to eventually build a Multi-terminal HVDC (MTDC) offshore grid.

In Europe, suggestions have been made to build a North Sea grid in order to transport the rising amount of power that goes together with the expansion of the offshore wind assets [3]. Also, the EU climate and energy package plans to raise the average renewable share in the EU to $20 \%$ by 2020 . The targets will contribute to decrease Europe's dependence on imported energy and to reduce greenhouse gas emissions [4]. Since VSC HVDC combines the advantages of low transport losses and no cable charging current with

\footnotetext{
*Corresponding author. Tel.: +32163210 20, Fax.: +3216321985.

Email addresses: kailash.srivastava@se.abb.com (K. Srivastava), muhamad.reza@se.abb.com (M. Reza), stijn.cole@esat.kuleuven.be (S. Cole), jef.beerten@esat.kuleuven.be (J. Beerten ), ronnie.belmans@esat.kuleuven.be (R. Belmans) 
the ability to easily operate multiple buses in parallel, it is likely to become part of the solution [3]. The UK National Grid foresees significant further development of wind generation. There are indications for a large growth in large off-shore wind farms rising from $140 \mathrm{MW}$ in 2006/07 to $3445 \mathrm{MW}$ by 2012/13 [5]. The test model used in this paper is a possible first stage towards a North Sea Supergrid [6], with two non-synchronized AC systems (e.g. wind farms) connected to the UK grid by a DC network.

In literature, most VSC MTDC configurations consist of one bus regulating the DC voltage (slack bus) and a set of other DC buses, whose converters regulate the active power injected in the AC grid. The slack bus ensures that the total amount of active power going into the grid equals the sum of the amount of power going out plus the losses in the DC system. It performs this task by controlling the DC voltage to its reference value. Basically, there is no need for external communication since a voltage measurement suffices for the slack bus to adjust its active power properly. As long as the power required to keep the DC network in a stable operating point does not exceed the power rating of the slack converter and the DC voltage control is fast enough to handle severe transients, DC overvoltages can be avoided. However, when this is not the case, transients will put a lot of stress on the slack bus and it will no longer succeed in keeping the voltage within limits.

A solution to this problem is a distributed slack bus. Active power regulating buses help the original slack bus (main slack bus) in keeping the DC voltage stable. There are two options. The first one is generally known as the voltage margin method and is described in [7]. It ensures that the DC voltage is controlled around a new reference value by another converter once a DC voltage margin has been exceeded. This happens when the main slack bus can no longer supply or extract the power necessary to control its DC bus voltage. This control strategy has the disadvantage that the voltage margin needs to be large, in order to avoid unstable interactions between the converters [8]. The second option is to implement a DC voltage droop, as illustrated in [8-10]. In this approach, the power setting of the slack bus is linked with the variation of the DC voltage. This method allows to regulate the DC voltage on several buses at the same time. This paper investigates a control strategy where a proportional-integral (PI) control on the main slack bus is combined with a proportional (droop) control on the other buses. It was already shown in [10] that this is a feasible option when the power rating of the main slack bus is reached. Therefore this paper will focus on improvements in transient stability, based on the work presented in [11]. In this paper, the modeling of the $P, Q$ and $V_{D C}$ controls is discussed. Next, $P-V_{D C}$-characteristics illustrate how these controls are applied in both the voltage margin method and voltage droop method. A strategy that combines one PI- $V_{D C}$-controller with one or more proportional $V_{D C}$-controllers on other buses is explained. Finally, the positive effect on the transient behavior is shown by simulation results. 


\section{VSC Control}

\subsection{Outer Controller}

The voltage on the grid side of the phase reactor, is called the filter bus voltage and denoted $V_{f}$. The current flowing though the phase reactor is denoted $I$. When transforming both quantities to a synchronously rotating $d q$-reference frame, the expressions for the active and reactive power at the filter bus become:

$$
\begin{aligned}
& P=V_{f, d} I_{d}+V_{f, q} I_{q}, \\
& Q=V_{f, q} I_{d}-V_{f, d} I_{q} .
\end{aligned}
$$

Since $V_{f, q}$ is zero when the $d$-axis is aligned with $V_{f}, I_{d}$ and $I_{q}$ can respectively be used to control the active and reactive power. The resulting controllers are shown in Figs. 1a and 1b. The DC voltage, $V_{D C}$, is controlled by balancing the active power injections into the DC grid. As a consequence, the DC voltage controller, shown in Fig. 1c, also uses $I_{d}$ as a controlling quantity. The controller shown here also incorporates a feed-forward compensation as proposed in [9] and [12].

[Figure 1 about here.]

\subsection{Inner Controller \& Converter Model}

The inner current controller is a fast PI controller, tracking the current reference supplied by the outer controller. It is shown together with the converter model and phase reactor model in Fig. 2. In this figure:

$$
\begin{gathered}
C 1=\frac{K_{p} s+K_{i}}{s}, \\
C 2=\frac{1}{1+T_{w} s}, \\
C 3=\frac{1}{s L_{P R}+R_{P R}}, \text { and } \\
C 4=\omega L_{P R} .
\end{gathered}
$$

[Figure 2 about here.]

This is the same model as was proposed in [9]. The output of the inner coupled current controllers is the reference converter voltage $V_{c, r e f}$, which is used to synthesize the converter voltage reference. The actual converter voltage lags the reference voltage due to the time-lag of the converters power electronics. As discussed in [13], a time delay can be added to represent these fast converter dynamics. More detailed converter models can be considered when the control parameters are fine-tuned. Both approaches result in a similar overall system response, as discussed in [13]. When the actual converter voltage $V_{c}$ is applied to the phase reactor, this results in the converter current $I$. As described above, the VSC is controlled at its 
converter side, using the quantities at the grid side as input signals. When assuming the converter to be lossless, the impact on the DC side is found using (3)-(5).

$$
\begin{gathered}
P_{c}=V_{c, d} I_{d}+V_{c, q} I_{q}, \\
I_{D C, c}=\sum_{i=1}^{n} I_{D C, i}, \\
\frac{d V_{D C}}{d t}=\frac{\frac{P_{c}}{2 V_{D C}}-I_{D C, c}}{C_{c o n v}+\sum_{i=1}^{n} \frac{C_{\text {line }, i}}{4}},
\end{gathered}
$$

with $P_{c}$ the active power measured at the filter bus and $I_{D C}$ the current through a DC cable, flowing away from the converter. The converter DC capacitor $C_{c o n v}$ and lumped cable capacitance $C_{\text {line }}$ are as shown in Fig. 3.

[Figure 3 about here.]

\section{Voltage Margin Control}

\subsection{Point to Point HVDC Transmission}

The $P-V_{D C}$ characteristic of one bus under voltage margin control is presented in Fig. 4a. As shown in this figure, the bus will act as the DC voltage regulator as long as its active power injection $P$ is not reaching the power limits. Once $P$ reaches these limits, it will act as an active power controller and regulate $P$ to the limit value. With the slack bus inverting power at its lower limit $P_{\min }$, it will not be able to stop $V_{D C}$ from rising above the reference value. The opposite is true for the active power limit on the rectifier side.

Fig. 4b shows how two buses with this kind of characteristic can be combined. A stable operating point is obtained where a power regulation line of one bus crosses the voltage regulation line of the other bus. In order to avoid unfavorable interactions between the voltage controls at both ends, a voltage margin is introduced between the reference voltages of both buses [7]. This ensures that during small fluctuations in DC voltage only one bus acts as a voltage regulator. In essence this is nothing more than the standard control, i.e. there is one DC voltage regulating bus and one active power regulating bus. According to [7], there are two strategies in which the operating point, and thus the power transmitted, can be adjusted. The first and most straightforward way is by adjusting the power limit. This is shown in Fig. 4c. The other way is by changing the reference voltage, as shown in Fig. 4d. In this way, the former active power dispatching bus becomes the DC voltage regulating bus and the former DC voltage regulating bus is now free to control the amount of power to be transmitted. The advantage of this approach is that both characteristics are 
crossing the $\mathrm{P}=0$ line. This implies that if one bus would be suddenly stopped, the other one can maintain stable operation and it can still operate as a STATCOM.

[Figure 4 about here.]

\subsection{Multi-Terminal HVDC Transmission}

In a multi-terminal configuration, the same control can be used. To reduce the need for communication even further, [7] presents a two-stage DC voltage control. The main slack converter is still implemented as before with its voltage reference at $V_{D C, r e f}$. The other converters will be implemented in two stages, as shown in Fig. 5. This increases the flexibility in two ways. First, the active power in all buses can be easily regulated by changing $P_{\text {ref }}$. At the same time the active power limits $P_{\min }$ and $P_{\max }$ can be kept at the physical boundaries of the converter at all times. Second, it provides an easy way to give all buses a certain level of importance. For example, if it is desired that one bus always keeps working at $P_{r e f}$, both the upper and lower voltage margin can be made sufficiently large. In that way, other buses will be assigned the role of slack bus first, when the main slack bus has reached its limits.

A PI-controller is a straightforward choice to regulate $V_{D C}$, since it will always control the DC voltage to its reference value. However, it should be noted that it is not feasible to control $V_{D C}$ at different places at the same time. This could give rise to suboptimal operating points (statically) [11] and hunting oscillations (dynamically) [8]. Instead, voltage droop control needs to be used in this case.

[Figure 5 about here.]

\section{Voltage Droop Control}

When voltage droop control is used in the absence of a PI-controller, the voltage controller's active power $P$ will change when the value of the DC bus voltage $V_{D C}$ changes. This principle is shown in Fig. 6 . When $V_{D C}$ drops (e.g. due to large withdrawal of power someplace else in the DC network) the slack bus will increase the active power injection in the DC grid $P$ until a new equilibrium point, at a lower DC voltage, is reached. The use of a proportional $(\mathrm{P}) V_{D C}$-controller allows multiple converters to regulate the voltage at the same time.

This control can now be adapted by including a voltage margin. The voltage droop characteristic with a voltage margin is shown in Fig. 7. This characteristic can be horizontally transposed when $P_{r e f}$ needs to be changed. Note however that there should always be one converter (the main slack converter) implemented with a voltage droop without this voltage margin, or with a PI-controller. This will give the best transient response since it allows a continuous regulation of $V_{D C}$ around $V_{\text {ref }}$. When the main slack converter also uses droop control, the other converters join in regulating $V_{D C}$, once the voltage margin is exceeded. When 
the main slack converter is using a PI-controller to regulate $V_{D C}$, the droop on the other buses will only have effect in case of transients or in case the voltage drop across the DC cables is larger than the voltage margin. This is obvious since the PI-controller will ensure there is no steady state error and therefore $V_{D C}$ will stay within the voltage margin. A PI-controller on the main slack bus is preferable because it lowers the voltage rating of the converters.

[Figure 6 about here.]

[Figure 7 about here.]

\section{Simulation Studies}

\subsection{Test Model}

The test model used is shown in Fig. 8. It is based on the idea of connecting several non-synchronized AC systems (e.g. offshore wind farms) with the United Kingdom using VSC HVDC transmission. This DC network can also alleviate congestion on the AC grid and gradually evolve towards a North Sea grid. In the AC grid, the electricity consumption is situated mainly in the south, around London, while a lot of production is situated in the north. The generators were modelled with the well-known classical generator model (Model 0.0), as defined in [14]. The generators and have a combined generating capacity of $27 \mathrm{GW}$. The resulting short circuit powers are 51 GVA for the slack bus (in the north), and 29 GVA for grid bus 2 (in the south). Bus 3 and 4 are modelled as infinite buses. The parameter values used in the simulations are summarized in table 1.

[Figure 8 about here.]

[Table 1 about here.]

\subsection{Results}

The simulations investigate to what extent a voltage droop control on grid bus 2 can assist the main slack bus in handling transients. The proposed system has been implemented in MatDyn, a Matlab-based toolbox for power system dynamic simulation [15]. The loss of $300 \mathrm{MW}$ generation at bus 3 was chosen to investigate the transient response of the controllers. The results are shown in Figs. 9 and 10. In Fig. 9 , the dashed lines show the system's response with only one voltage controller operating. The full lines represent the response with voltage droop control present on grid bus 2. The voltage droop is triggered at a DC voltage of $0.97 \mathrm{pu}$. It is clear that the transient overvoltages and undervoltages are reduced. The main drawback is the sudden change in active power, as shown in Fig. 10. Grid bus 2 will even turn into a rectifier for a short amount of time. In the simulations this results in an AC voltage drop at grid bus 2 of $9 \%$. This might be a problem in many AC systems. 
It was found that the oscillations on $P, V_{D C}$ and $V_{A C}$ can be damped by lowering the voltage margin. This is shown in Figs. 11 and 12. A compromise has to be made, since lowering the voltage margin might result in a static operating point on the droop, when the voltage drop across the DC cables is sufficiently large. This is clear from Fig. 12. The power of grid bus 2 does not fully return to its original value, because the new stable operating point is situated on the droop characteristic. The oscillations are clearly damped: both $P$ and $V_{D C}$ oscillations are smaller and shorter, and the AC voltage drop on grid bus 2 now only drops with less than $5 \%$. However, an operating point on the droop characteristic might cause oscillations when the droop characteristic is horizontally transposed in the $P-V_{D C}$ plane. This happens when $P_{r e f}$ is changed. Therefore this situation should be avoided if no additional control, e.g. an additional PI controller for $P_{\text {ref }}$, is in place. This control should also incorporate a compensation for the deviation from the operating point to $P_{\text {ref }}$.

[Figure 9 about here.]

[Figure 10 about here.]

[Figure 11 about here.]

[Figure 12 about here.]

\section{Conclusion}

Extended MTDC systems are likely to be built in the future and a distributed voltage control is recommended to reduce the dependence on the main slack bus. A DC voltage control strategy has been suggested with one PI-controller on the main slack bus, combined with a voltage droop characteristic on at least one other DC bus. The simulations show that DC voltage transients can be significantly reduced by applying this control structure. The impact on the AC grid should always be kept in mind. Adjusting the voltage margin provides one of the means to alter this impact.

\section{Acknowledgement}

Jef Beerten is funded by a research grant from the Research Foundation - Flanders (FWO).

\section{References}

[1] ABB, It's time to connect, available online: http://library.abb.com/global/scot/scot221.nsf/veritydisplay/ bcd2f0a98218a66bc1257472004b83a8 (sept 2008).

[2] B. Andersen, VSC Transmission, Tech. rep., CIGRÉ, Working Group B4.37 (2005).

[3] J. Blau, Europe plans a North Sea grid, Spectrum, IEEE 47 (3) (2010) 12 -13. doi:10.1109/MSPEC.2010.5421884. 
[4] European Commission, The EU climate and energy package, URL: http://ec.europa.eu/environment/climat/climate_ action.htm, last checked on 2010-04-18.

[5] National Grid, GB Seven Year Statement, URL: http://www.nationalgrid.com/uk/sys_06, last checked on 2010-04-07.

[6] Friends of the Supergrid, Supergrid, URL: http://www.friendsofthesupergrid.eu, last checked on 2010-04-07.

[7] T. Nakajima, S. Irokawa, A control system for HVDC transmission by voltage sourced converters, in: Power Engineering Society Summer Meeting, 1999. IEEE, Vol. 2, 1999, pp. 1113 -1119 vol.2. doi:10.1109/PESS.1999.787474.

[8] R. Hendriks, G. Paap, W. Kling, Control of a multi-terminal VSC transmission scheme for connecting offshore wind farms, in: European Wind Energy Conference \& Exhibition, 2007.

[9] T. Haileselassie, K. Uhlen, T. Undeland, Control of Multiterminal HVDC Transmission for Offshore Wind Energy, in: Nordic Wind Power Conference Presentation, 2009.

[10] L. Xu, B. Williams, L. Yao, Multi-terminal DC transmission systems for connecting large offshore wind farms, in: Power and Energy Society General Meeting - Conversion and Delivery of Electrical Energy in the 21st Century, 2008 IEEE, 2008, pp. 1 -7. doi:10.1109/PES.2008.4596508.

[11] C. Dierckxsens, VSC MTDC Systems with Distributed Slack Bus, Master's thesis, Katholieke Universiteit Leuven, Belgium (2010).

[12] S. Ruihua, Z. Chao, L. Ruomei, Z. Xiaoxin, VSCs based HVDC and its control strategy, in: Transmission and Distribution Conference and Exhibition: Asia and Pacific, 2005 IEEE/PES, 2005, pp. 1 -6. doi:10.1109/TDC.2005.1546800.

[13] S. Cole, J. Beerten, R. Belmans, Generalized dynamic VSC MTDC model for power system stability studies, IEEE Trans. on Power Systems 25 (3) (2010) 1655-1662.

[14] IEEE Task Force, Current usage \& suggested practices in power system stability simulations for synchronous machines, IEEE Trans. on Energy Conversion EC-1 (1) (1986) 77-93. doi:10.1109/TEC.1986.4765673.

[15] S. Cole, R. Belmans, Matdyn, a new matlab-based toolbox for power system dynamic simulation, IEEE Trans. on Power Systems (99) (2010) 1-8, early Access. doi:10.1109/TPWRS.2010.2071888. 


\section{List of Figures}

1 Common outer control loops . . . . . . . . . . . . . . . . . . . . . . . 10

2 The inner current loop, converter, and phase reactor model [9]. . . . . . . . . . . . . . 11

3 DC side of the converter and a $\pi$ section of a DC line. . . . . . . . . . . . . . . . . 12

5 Voltage margin characteristic for multi-terminal HVDC [7] . . . . . . . . . . . . . . . . . 14

6 Voltage droop characteristic [8] . . . . . . . . . . . . . . . . . . . . . 15

$7 \quad$ Voltage droop characteristic with voltage margin. . . . . . . . . . . . . . . . . . . 16

8 DC test model. . . . . . . . . . . . . . . . . . . . . . . . . . . 17

9 DC voltages. . . . . . . . . . . . . . . . . . . . . . . . . . . 18

10 Active powers. . . . . . . . . . . . . . . . . . . . . . 19

11 DC voltages, operating point on droop characteristic after transient. . . . . . . . . . . . . 20

12 Active powers, operating point on droop characteristic after transient. . . . . . . . . . . . 21 


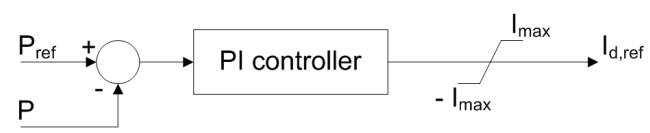

(a)

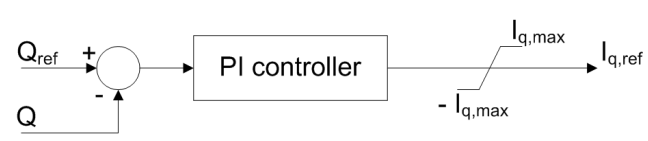

(b)

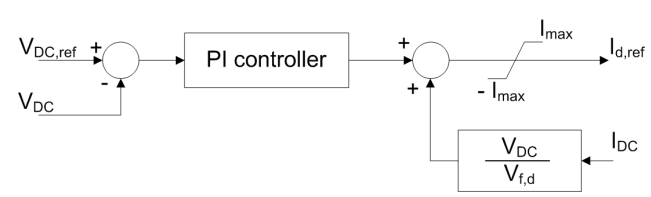

(c)

Figure 1: Common outer control loops: (a) active power control; (b) reactive power control; and, (c) DC voltage control [9]. 


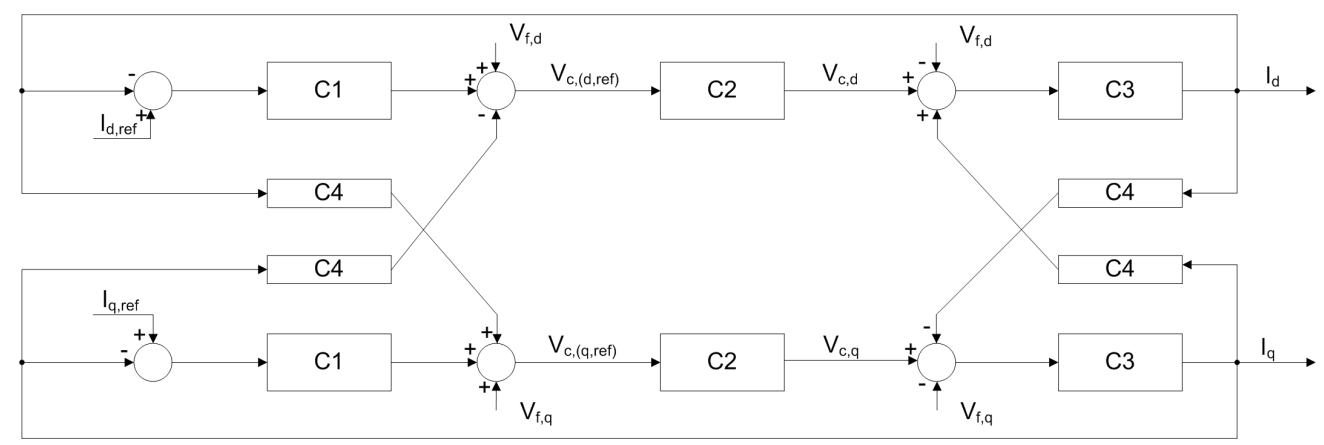

Figure 2: The inner current loop, converter, and phase reactor model [9]. 


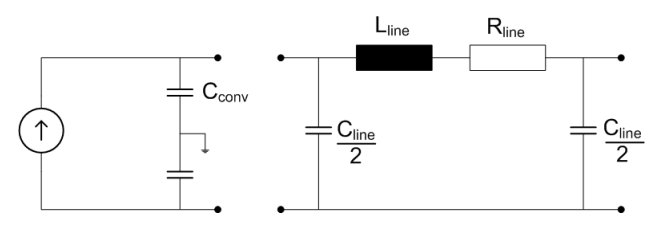

Figure 3: DC side of the converter and a $\pi$ section of a DC line. 


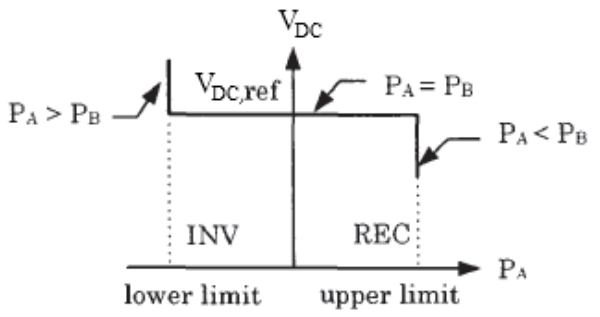

(a)

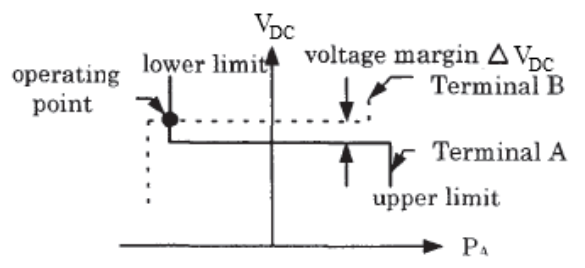

(b)

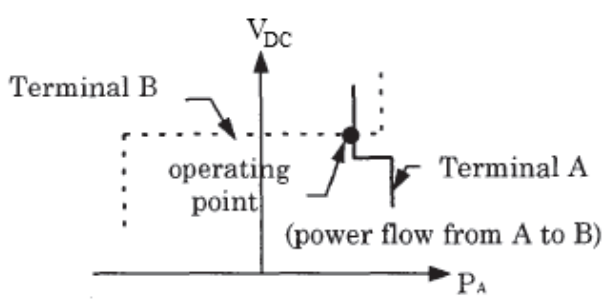

(c)

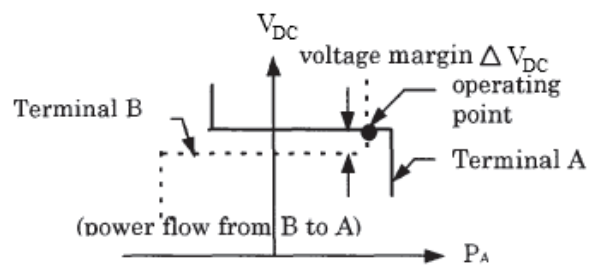

(d)

Figure 4: Voltage margin method illustration [7]: (a) characteristic of one converter; (b) operating point before power reversal; and, (c)-(d) after power reversal. 


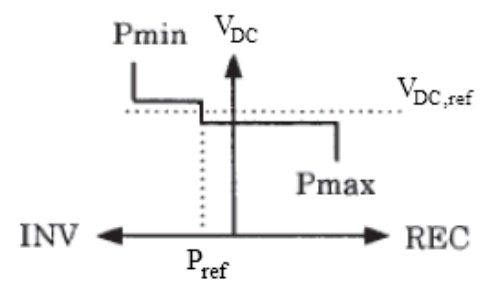

Figure 5: Voltage margin characteristic for multi-terminal HVDC [7]. 


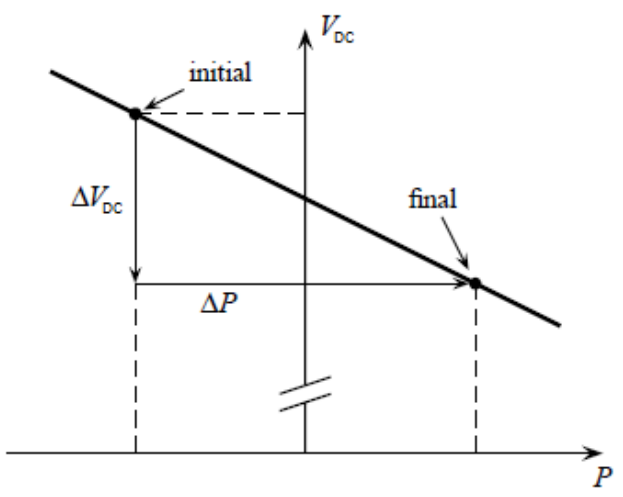

Figure 6: Voltage droop characteristic [8]. 


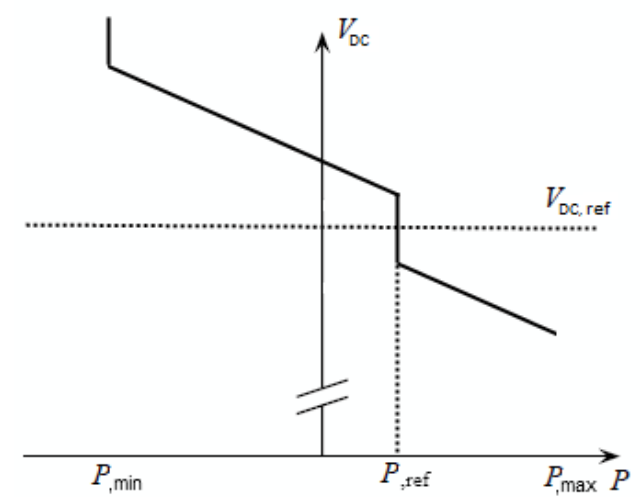

Figure 7: Voltage droop characteristic with voltage margin. 


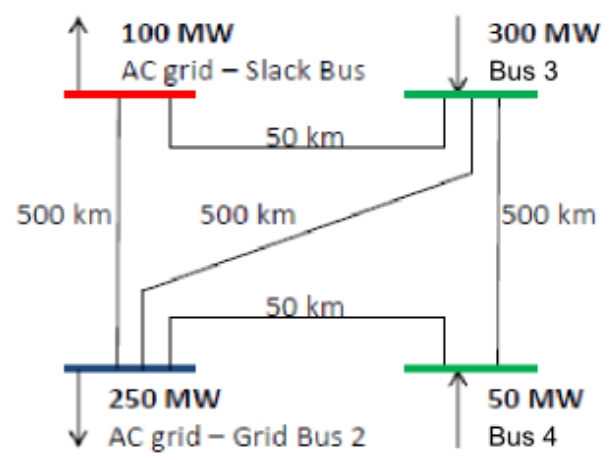

Figure 8: DC test model. 


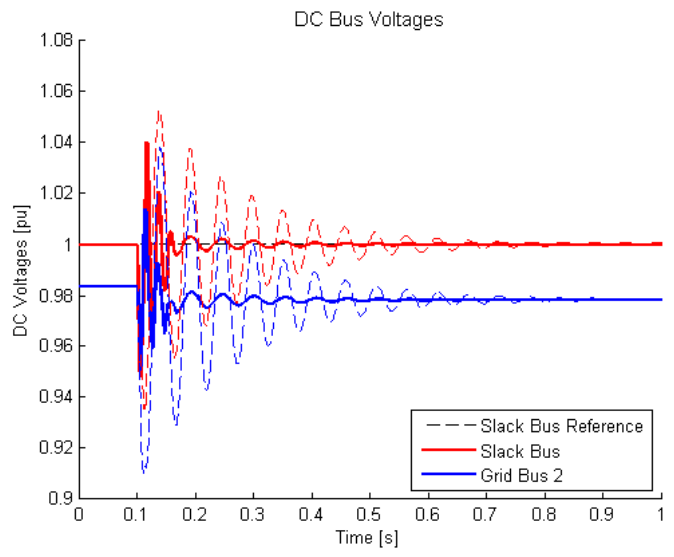

Figure 9: DC voltages. 


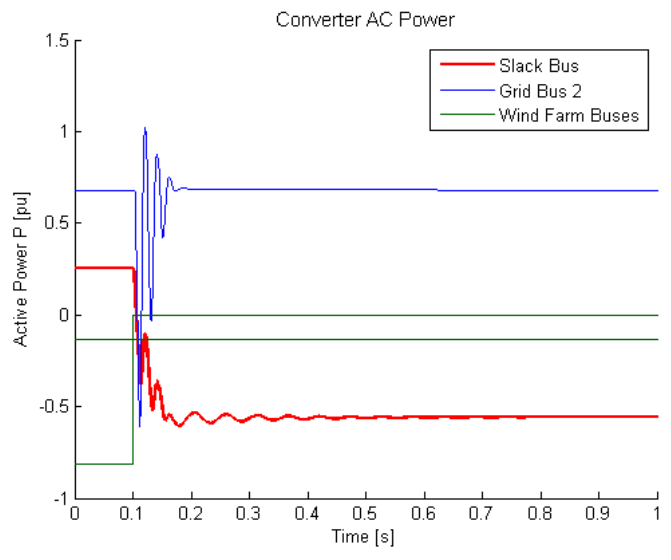

Figure 10: Active powers. 


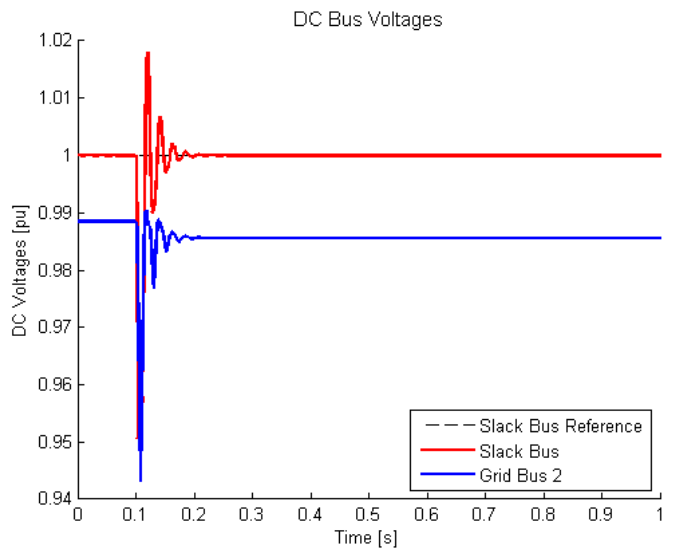

Figure 11: DC voltages, operating point on droop characteristic after transient. 


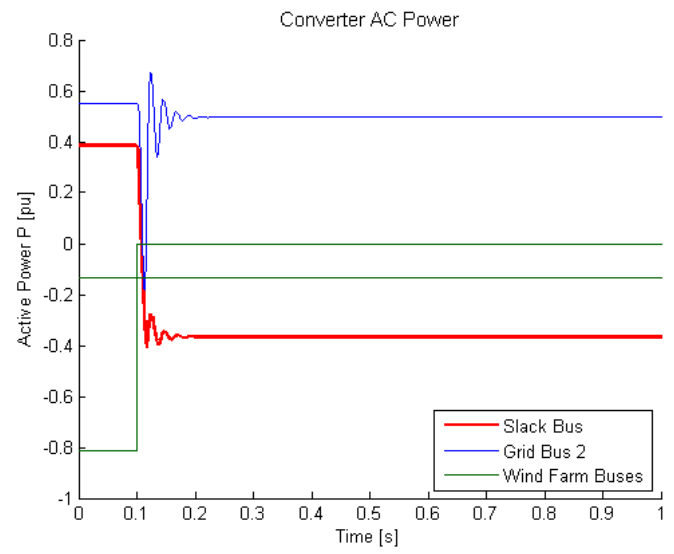

Figure 12: Active powers, operating point on droop characteristic after transient. 


\section{List of Tables}

1 Model parameter values . . . . . . . . . . . . . . . . . . . . . . . . . 23 
Table 1: Model parameter values

\begin{tabular}{lcl}
\hline DC cable & & \\
\hline$R_{\text {line }}$ & $2.4 e-2$ & $\Omega / k m$ \\
$L_{\text {line }}$ & $2.1 e-4$ & $H / k m$ \\
$C_{\text {line }}$ & $5.92 e-7$ & $F / k m$ \\
\hline Converter & & \\
\hline$P_{\text {conv }, N}$ & 350 & $M W$ \\
$C_{\text {conv }}$ & $62.7 e-6$ & $F$ \\
$T_{w}$ & 0.002 & $s$ \\
\hline Phase Reactor and transformer & & \\
\hline$S_{T R, N}$ & 380 & $M V A$ \\
$X_{P R}$ & 17.3 & $\Omega$ \\
$R_{P R}$ & 0.1 & $\Omega$ \\
$X_{T R}$ & 13.0 & $\Omega$ \\
\hline
\end{tabular}

